\title{
A study on exoenzyme activities of candida albicans isolated from oral cavities of hiv-infected patients on HAART
}

\begin{abstract}
The mortality and morbidity of HIV-infected patients have declined with the advent of highly active antiretroviral therapy (HAART), but its effect on Candida infections remains elusive. The aims of the present study were to determine the effect of HAART on the phosphor lipase, proteinase and haemoltyic activities of oral Candida albicans, and to investigate the correlation between these enzyme profiles and the patients' demographic and clinical parameters. Isolates were obtained from 16 Chinese HIV-infected patients on HAART and 16 healthy controls using oral rinse technique. The isolates were tested in vitro for phospholipase, proteinase and haemolytic activities using three different plate assays. The phospholipase and haemolytic activities were found to be significantly higher $(p<0.05)$ and the proteinase activities were lower in the test group $(p<0.05)$. The lower proteinase activities in HIV-infected group could be due to the direct effect of protease inhibitors in HAART while the higher phospholipase and haemolytic activity in the HIVinfected group remains elusive. These finding provide evidence of altered extracellular enzyme activities in the Candida isolates obtained from HIV patients on HAART, which may affect the infectivity of the Candida isolates.
\end{abstract}

Keywords: Highly active anti-retroviral therapy (HAART), Candida albicans, Phospholipase, Proteinase, Haemolysin
Volume 2 Issue 2 - 2015

\author{
Shibu Thomas, ' CSP Tsang,' Asok Mathew, \\ Sajith Bhaskar,' Sivan Padma Priya,' Nisha \\ Varughese ${ }^{3}$ \\ 'College of dentistry,Ajman University of science and \\ technology AI Fujairah, UAE \\ 2Prince Philip Dental Hospital, University of Hong Kong, China \\ ${ }^{3}$ Department of orthodontics, Dubai College of dental medicine, \\ UAE
}

Correspondence: Asok Mathew, Faculty, College of dentistry, Ajman University of science and technology AI Fujairah, UAE, Tel 0097।503260639,Email drashokm@gmail.com

Received: January 24, 2015 | Published: March 09, 2015
Abbreviations: HAART, Highly Active Anti-Retroviral Therapy; HIV-PIs, HIV-Protease Inhibitors; SAP, Secreted Aspartyl Proteinase; PBS, Phosphate Buffered Saline; SDA, Sabouraud Dextrose Agar; BSA, Bovine Serum Albumin; NNRTI, NonNucleoside Reverse Transcriptase Inhibitors; NRTI, Nucleoside Reverse Transcriptase Inhibitors

\section{Introduction}

Morbidity and mortality have decreased significantly since the introduction of highly active anti-retroviral therapy (HAART) for treating HIV-infected patients. ${ }^{1,2}$ The decline in the incidence of oro pharyngeal candidiasis is thought to be due to immune reconstitution of the host and HIV-protease inhibitors (HIV-PIs) in HAART. HIV-PI has been shown to exert both in vivo and in vitro effects on Candida species; in particular, the inhibition of secreted aspartyl proteinase (Sap) secretion. ${ }^{3,4}$

Saps are encoded by 10 SAP genes and different genes appear to play different roles in C. albicansinfections. These genes are involved in adhesion, tissue damage, and evasion of host immune responses. ${ }^{5}$ In the pre-HAART era, Candida isolated from HIV-infected patients was found to have higher proteolytic activities. ${ }^{6,7}$ Although HIVPI demonstrates a protective effect against candidal infection, De Bernardis et al. ${ }^{8}$ found an increase in Sap production in vitro from sequential isolates of $C$. albicans from patients on HAART-PIs. ${ }^{8}$ Therefore it is considered that other factors may also be involved in decreasing the incidence of oro pharyngeal candidiasis in HIVinfected patients.

Besides Saps, other exo enzymes such as phospholipases may also play an important role in the pathogenesis of Candida. Phospholipases are important in catalyzing the hydrolysis of phospholipids which are the major components of host cell membranes to thereby facilitate candidal invasion. ${ }^{9}$ Seven phospholipase genes (PLA, PLB1, PLB2,
PLC1, PLC2, PLC3 and PLD1) have been identified, ${ }^{10}$ of which only four of them (PLB1, PLB2, PLC1 and PLD1) have been well characterized ${ }^{11-14}$ Limited studies have been conducted on PLA, PLC2 and PLC3 are not critical for morphogenesis and interaction with macrophages and their roles in pathogenesis have not been fully elucidated. ${ }^{15}$

Haemolysin is another putative virulence factor that contributes to candidal pathogenesis. C. albicans have the ability to secrete haemolysin to lyse host erythrocytes and strip iron from hemoglobin molecules, which facilitates hyphal invasion in disseminated candidacies. ${ }^{16}$ A partial DNA sequence of HLPalso known as putative haemolysin gene, encoding a protein with haemolytic activity in Candida was amplified and cloned. ${ }^{17}$ Subsequently Luo G et al. ${ }^{18}$ demonstrated the HLP gene expression in C. glabrata, to illustrate the role of HLPgene in haemolysis.

To date, only a few studies have reported on the phospholipases and haemolysin activities in HIV-infected individuals. The effect of HAART-PI on C. albicanssecretion or activities, and their relations with Saps are still unknown. Therefore, the aims of the present study were to determine the Sap, phospholipase and haemolysin activities of $C$. albicans isolated from HIV-infected patients on HAART, compare it with a healthy control group and to investigate if there is any correlation between the enzyme profiles of $C$. albicans isolates and patients' demographic data, stage of HIV infection, risk for HIV, current medications, CD4 counts, HIV viral loads, salivary $\mathrm{pH}$ and history of oral candidiasis.

\section{Materials and methods}

Sixteen oral Candida Albicansisolates obtained from HIV-1 infected Chinese patients who were recruited from the Special Medical Services, Department of Medicine, Queen Elizabeth Hospital, Hong Kong SAR, China were used in the study. The inclusion criteria 
of the patients were HIV-infected and aged 18 years or above who were stabilized under HAART for at least one year. Subjects who had received antifungals or pentamidine during the last six months preceding the examination, those with a history of acute systemic disease, such as fever or diarrhea, and those who were smokers or pregnant were excluded. An equal number of healthy individuals, age and sex matched were to constitute the control group. Ethical Approval was obtained from the Institutional Review Board of the University of Hong Kong and the Kowloon central cluster. Informed consent was obtained prior to study procedures.

A modified protocol for concentrated rinse culture was used to isolate $C$. albicans from the patients. ${ }^{19}$ In brief, patients were given $10 \mathrm{ml}$ phosphate buffered saline (PBS) $(0.1 \mathrm{M}, \mathrm{pH} 7.3)$ in a sterile universal container and instructed to rinse the mouth for $60 \mathrm{~s}$. After the oral rinse was expectorated into the container, the sample was transferred immediately to the laboratory, where it was centrifuged at $1700 \mathrm{~g}$ for 10 minutes. The supernatant was discarded and the pellet re suspended in $2 \mathrm{ml}$ PBS on a vortex mixer for $30 \mathrm{~s}$. A spiral plater (Model DU; spiral system) was used to dispense $50 \mathrm{ml}$ of the suspension onto various media, as described below.

The concentrated oral rinse was spiral-plated with an Archimedean spiral onto a Sabouraud dextrose agar (SDA) plate (Gibco) and incubated for $48 \mathrm{~h}$ at $37^{\circ} \mathrm{C}$. Well-separated yeast colonies were sub cultured onto SDA plates to obtain pure yeast cultures that were then harvested suspended in water in sterile vials and stored at $-20^{\circ} \mathrm{C}$. All yeast isolates were identified with a commercially available identifying kit system, ID32C (bioMerieux).

\section{Data collection}

Demographic data and information on education level, income and presence of any extra-oral or intra-oral lesion of the patients was gathered using a researcher-administered questionnaire. The HIV1 viral load, CD4+ cell counts, risk for HIV, HIV staging, current medications and history of oral candidiasis were recorded from the medical files.

\section{Determination of phospholipase activity}

Extracellular phospholipase activity of $C$. albicansisolates was screened by measuring the size of precipitation zone on egg yolk agar after growth. ${ }^{10}$ The medium of egg yolk agar contained of $0.11 \mathrm{~g} \mathrm{CaCl}_{2}$, $11.7 \mathrm{~g} \mathrm{NaCl}, 13.0 \mathrm{~g}$ SDA (Gibco) and 10\% sterile egg yolk (Oxoid) (with in $184 \mathrm{ml}$ distilled water). Initially, the constituents devoid of the egg yolk were mixed and sterilized, followed by egg yolk was centrifuged at $500 \mathrm{~g}$ for $10 \mathrm{~min}$ at room temperature and addition of $20 \mathrm{ml}$ of the supernatant was done to the sterilized medium. Test and control Candida isolates of standard inoculate $\left(10 \mathrm{ul}\right.$, with $10^{8}$ yeast cells per $\mathrm{ml}$ saline) were deposited onto the egg yolk agar medium and room temperature was used to dry it. Diameter of the precipitation zone around the colony was determined of each culture after their incubation at $37^{\circ} \mathrm{C}$ for $48 \mathrm{~h}$. (phospholipase activity indicator).

The Reading of the plates were done using computerized image analysis system (Quantimet 500 Qwin; Leica), that measured the diameter of the colonies on a magnified scale relative to the precipitation zones. The ratio of the diameter of the colony to the diameter of the colony plus the precipitation zone $(\mathrm{mm})$ was expressed as Phospholipase activity (PZ value).$^{20}$ Each yeast isolate was tested in duplication on three separate occasions. Positive control used was reference strains of C. albicans (ATCC 90028) and Candida glabrata (90030) served as a negative control, from our laboratory stock collection.

\section{Determination of proteinase activity}

Analysis of extracellular proteinase activity of C. albicans strains were done in terms of bovine serum albumin (BSA) degradation conferring to the technique described by Staib (1965). ${ }^{21}$ In short, a suspension of $1 \times 10^{6}$ cells $\mathrm{ml}^{-1}$ from $18 \mathrm{hrs}$ was prepared, and $1 \%$ BSA plate was used to inoculate $10 \mathrm{ml}$ of suspension. Incubation of plate was done for 5 days at $37^{\circ} \mathrm{C}, 1.25 \%$ naphthalene black solution was flooded for $15 \mathrm{~min}$ and washed with $90 \%(\mathrm{~V} / \mathrm{V})$ methanol/water distaining solution. Decolonization for $36 \mathrm{~h}$ followed by several changes of distaining solution.

The Reading of the plates were done using computerized image analysis system (Quantimet 500 Qwin; Leica), that measured the diameter of the colonies on a magnified scale relative to the precipitation zones. The ratio of the diameter of the colony to the diameter of the clear zone of proteolysis $(\mathrm{mm})$ was expressed as Proteinase activity $\left(\mathrm{P}_{\mathrm{rz}}\right.$ value). Each yeast isolate tested in duplication on three separate occasions. Positive control used was reference strains of C. albicans (ATCC 90028) and Candida parapsilosis ATCC 22019 served as a negative control.

\section{Determination of haemolysin activity}

Blood plate assay was used to evaluate haemolysin activity. ${ }^{22,23}$ By adding $7 \mathrm{ml}$ fresh sheep blood (Dixon) to $100 \mathrm{ml}$ SDA (Gibco) supplemented with $3 \%(\mathrm{w} / \mathrm{v})$ of glucose as a final concentration the media was prepared. The final medium $\mathrm{pH}($ mean $\pm \mathrm{SD})$ was $5.6 \pm$ 0.2 . The medium was deposited with standard inoculum from each of the test and control Candida isolates $\left(10 \mathrm{ml}\right.$, with $10^{8}$ yeast cells per $\mathrm{ml}$ saline). The plates for $48 \mathrm{hrs}$ were incubated at $37^{\circ} \mathrm{C}$ in $5 \% \mathrm{CO}_{2}$.

The Reading of the plates were done using computerized image analysis system (Quantimet 500 Qwin; Leica), that measured the diameter of the colonies on a magnified scale relative to the precipitation zones. The ratio of the diameter of the colony to the diameter of the clear zone of haemolysis $(\mathrm{mm})$ was expressed as haemolytic activity ( $\mathrm{Hz}$ value). Each yeast isolate tested in duplication on three separate occasions. Positive control used was reference strains of C. albicans (ATCC 90028) and Candida parapsilosis ATCC 22019 served as a negative control.

\section{Results and discussion}

\section{Statistical analysis}

Data were analyzed using statistical analysis computer software (SPSS 15.0 for Windows $\mathbb{C}$, SPSS Inc, U.S.A), and normality tests were carried out using Kolmogorov-Smirnov test. Simple regression was used to analyzePz, $\mathrm{PrZ}$ and $\mathrm{Hz}$ by thegender, age, education level, income, $\mathrm{pH}$, risk for HIV, $\mathrm{CDC}$, current medications, duration on HAART, oral candidiasis, viral load, $\mathrm{CD} 4$ count and salivary $\mathrm{pH}$. The level of significance was set at $p<0.05$.

Colonization and infection by Candida has been shown to have an important economic impact for critically ill patients, in addition to the morbidity and mortality associated with Candida infections in such patients. ${ }^{24}$

Current study was aimed to investigate the exoenzyme activities of phospholipase, proteinase and haemolysin secreted by C. albicans, isolated from HIV-infected patients on HAART. These enzymes were selected because they exhibit specificity towards candidal pathogenesis, particularly by facilitating the hyphal invasion seen in disseminated candidacies. ${ }^{25}$ 


\section{Phospholipase production by C. albicans}

The ability of $C$. albicans to produce phospholipase reflected an important pathogenic feature of this opportunistic fungal pathogen. Candida phospholipases are suspected to act as virulence factors, possibly by facilitating fungal access and adherence to epithelial cells, invasion of the host tissue, and interference with host defense mechanisms. ${ }^{26}$

Earlier studies have stated phospholipase activity in $30-100 \%$ ofCandida isolates from various sites. ${ }^{6,20}$ In the current study, all 16 isolates in the test group showed phospholipase activities according to plate assay. Our study also showed that HIV positive patients on HAART had increased phospholipase activities compared to HIV negative control group. The $\mathrm{Pz}$ value of $C$. albicans isolates range from 0.56 to 0.93 for the test group and from 0.72 to 0.88 for the control (Table 1) $(p<0.05)$. This finding agrees with those from using 239 oral and vaginal C. albicans strains from HIV positive patients that showed significantly higher quantities of phospholipase. ${ }^{27}$ Others have reported difference in exoenzyme phospholipase activity in Candida from different ecological sites or disease states.

Table I Phospholipase, proteinase and Haemolysin activities of oral C. albicans isolated from HIV-infected patients on HAART and non-HIV subjects. Results are expressed as mean $\pm \mathrm{SD}$

\begin{tabular}{llllll}
\hline Activity & HIV-infected group, $\mathbf{n = 1 6}$ & Non-HIV group, $\mathbf{n = 1 6}$ & Test & Statistic & $\mathbf{p}$ value \\
\hline Phospholipase(Pz) & $0.676 \pm 0.107$ & $0.782 \pm 0.049$ & $t$ & -3.59 & $0.00 \mathrm{I}$ \\
Proteinase(Prz) & $0.64 I \pm 0.074$ & $0.507 \pm 0.144$ & $t$ & $3.3 \mathrm{I}$ & 0.003 \\
Haemolysin(Hz) & $0.498 \pm 0.023$ & $0.667 \pm 0.052$ & $t$ & $-\mathrm{II} .79$ & 0.000 \\
\hline
\end{tabular}

No significant association was observed between phospholipase activities of $C$. albicans isolated from the test group and the demographic data, risk for HIV, CDC, current medication, duration on HAART, oral candidiasis, viral load and CD4 count. However, there was a significant correlation of the $\mathrm{pH}$ value of saliva with the phospholipase activities in the test group $(\mathrm{R} \mathrm{Sq}$ Linear $=0.594)$, Salivary $\mathrm{Ph}$. Salivary flow rate, wearing dentures, smoking habits and alcohol are some host factors associated with an increased oral carriage rate of Candida species. ${ }^{27} \mathrm{C}$. albicans isolates from patients having respiratory tract infections produced significantly greater amounts of phospholipase than those from blood was well documented in two recent publications. ${ }^{28,29}$ Study by Mukherjee PK, et al. ${ }^{30}$ showed that $\mathrm{pH}$ changes have an effect on phospholipase mRNA expression. ${ }^{30}$ Samaranayake $\mathrm{YH}$ et al. $^{31}$ also showed that phospholipase gene expression could be affected by the growth conditions. ${ }^{22}$ Thus $\mathrm{pH}$ changes in the oral niche of HIV-infected patients on HAART may alter the normal PL expression and may explain the altered phospholipase activities observed in the present study. These data in general indicate that the isolation site of C. albicans as well as the disease state of the patient may be an important factor in dictating phospholipase activity.

\section{Proteinase production by $\mathrm{C}$. albicans}

In the present study, all of the C. albicans isolates were Sap producers. These results are comparable to the study of. ${ }^{32}$ They observed $94.1 \%$ of C. albicansisolates from immuno compromised patients are Sap producers. High prevalence of proteolytic activity among $C$. albicans strains were also reported. ${ }^{2,33,34}$

The Prz values in the present study ranged from 0.52 to 0.75 for the test group and from 0.29 to 0.77 for the control group (Table 1). The mean proteinase activity of isolates from the test group $(0.64 \pm 0.07)$ was significantly lower than that of the control isolates $(0.51 \pm 0.14)$ $(p<0.05)$. One possible explanation for the lower proteinase activities could be the direct effect of proteinase inhibitors of HAART on Sap. Studies by Cassone A et al. \& De Bernardis F et al. ${ }^{8,35}$ showed the same results, which reported that patients on HAART-PI, but not HAART-non- nucleoside reverse transcriptase inhibitors (NNRTI) strongly inhibited Sap expression. ${ }^{4,8}$

No significant association was observed between proteinase activities of $C$. albicans isolates from HIV infected group and demographic data, saliva $\mathrm{pH}$, risk for HIV, CDC staging, duration on HAART, history of oral candidiasis, viral load and CD4 count. However, patients taking abacavir, a nucleoside reverse transcriptase inhibitor (NRTI), were found to have higher proteinase activities compared to the control group ( $p<0.05)$. Since NRTI is not known to have anti-proteinase properties, the reason of the increased proteinase activities remains elusive warrants further investigations. Since proteinase production by Candida especially $C$. albicans has been shown to depend on various factors, conditions and even stage of infection, ${ }^{36}$ proteinase assays have been recommended to be interpreted with caution. ${ }^{37}$

\section{Haemolysin production by C. albicans}

Survival and the ability to establish infection with in the mammalian host by pathogenic organisms depends on the ability to acquire elemental iron. ${ }^{3,38}$ Pathogens acquire this iron indirectly from commonly available iron-containing compounds such as hemoglobin, as there is primarily no free iron in human host. ${ }^{39}$ For this pathogen uses the enzyme haemolysin which helps to destroys the heme moiety and enables them to extract the elemental iron.

Studies on the activity of haemolysin in C. albicansare limited and to the best of our knowledge there are no studies on the activity of haemolysin from oral C. albicans isolated from HIV patients on HAART. In present study, all the C. albicans isolates were haemolysin producers. The $\mathrm{Hz}$ values ranged from 0.46 to 0.54 for the HIV infected group and from 0.60 to 0.79 for the control (Table 1). Higher haemolysin activities were detected in the test group $(0.50 \pm 0.23)$ than in the control $(0.67 \pm 0.05)$ isolate $(p<0.05)$. No significant association was observed between haemolysin activities of $C$. albicans isolates from test group and gender, age, education level, income, risk for HIV, CDC staging, duration on HAART, history oral candidiasis, viral load and CD4 count. However, haemolysin activities were significantly lower in patients taking abacavir $(p<0.05)$. Similarly the action of abacavir on haemolysin is unknown and further investigations are warranted to find out how abacavir affects both haemolysin and proteinase activities.

The use of PIs ritonavir and lopinavir was also found to be associated with decreased haemolysin activities $(p<0.05)$. This is an interesting finding as the main anti-candidal action of PI is believed to be its action against candidal Sap, this "anti-haemolysin" action may further explain its anti-candidal action. Again, further investigations are warranted.

\section{Conclusion}

All three exo enzymes tested were expressed by $C$. albicans isolates from both the HIV-infected on HAART and control group. Significantly, higher phospholipase and haemolysin activities of the $C$. 
albicans isolates were seen in the HIV-infected group compared to the control group, whereas the proteinase activity was significantly lower in HIV-infected on HAART.

Successful oral colonization of C. albicans in HIV-infected on HAART depends on degree of exo enzyme activity. The range of host factors affecting the eco system in which yeast resides controls the qualitative and quantitative variations in expression of array of extracellular enzyme secreted by $C$. albicans. In order to understand the natural history and host pathogen relationship better, advanced studies on simultaneous expression of $C$. albicans exo enzymes are urgently required.

\section{Acknowledgments}

We thank Drs. M Perera, P Li and MP Lee for providing the Candida albicansstrains and clinical data of the HIV-infected patients used in this study.

\section{Conflicts of interest}

None.

\section{References}

1. Palella FJ, Delaney KM, Moorman AC, et al. Declining morbidity and mortality among patients with advanced human immunodeficiency virus infection. New Eng J Med. 1998;338(13):853-860.

2. Brodt HR, Kamps BS, Gute P, et al. Change incidence of AIDS-defining illness in the era of antiretroviral therapy. AIDS. 1997;11(14):1731-1738

3. Bullen JJ. The significance of iron in infection. Rev. Infect Dis. 1981;3(6):1127-1138

4. Cassone A, De Bernardis F, Torosantucci A, et al. In vitro and vivo anticandidal activity of human immunodeficiency virus protease inhibitors. J Infect Dis. 1999;180(2):448-453.

5. Naglik JR, Challacombe SJ, Hube B. Candida albicans secreted aspartyl proteinases in virulence and pathogenesis. Microbiol Mol Biol Rev. 2003;67(3):400-428.

6. Wu T, Samaranayake LP, Cao BY, et al. In-vitro proteinase production by oral Candida albicans isolates from individuals with and without HIV infection and its attenuation by antimycotic agents. J Med Microbiol. 1996;44(4):311-316.

7. De Bernardis F, Boccanera M, Rainaldi L, et al. The secretion of aspartyl proteinase, a virulence enzyme, by isolates of Candida albicans from the oral cavity of HIV-infected subjects. Eur J Epidemiol. 1992;8(3):362367.

8. De Bernardis F, Tacconelli E, Mondello F, et al, Anti-retroviral therapy with protease inhibitors decreases virulence enzyme expression in vivo by Candida albicans without selection of avirulent fungus strains or decreasing their anti-mycotic susceptibility. FEMS Immunol Med Microbiol. 2004;41(1):27-34.

9. Jayatilake JA, Samaranayake YH, Samaranayake LP. An ultrastructural and a cytochemical study of candidal invasion of reconstituted human oral epithelium. J Oral Pathol Med. 2005;34(4):240-246.

10. Samaranayake LP, Raeside JM, MacFarlane TW. Factors affecting the phospholipase activity of Candida species in vitro. Sabouradia 1984;22(3):201-207.

11. Bennett DE, McCreary CE, Coleman DC. Genetic characterization of a phospholipase $\mathrm{C}$ gene from Candida albicans: presence of homologous sequences in Candida species other than Candida albicans. Micro biology. 1998;144(1):55-72.

12. Leidich SD, Ibrahim AS, Fu Y, et al. Cloning and disruption of caPLB1, a phospholipase $\mathrm{B}$ gene involved in the pathogenicity of Candida albicans. J Biol Chem. 1998;273(40):26078-26086.
13. Sugiyama Y, Nakashima S, Mirbod F, et al. Molecular cloning of a second phospholipase B gene, caPLB2 from Candida albicans. Med Mycol. 1999;37(1):61-67.

14. Hube B, Hess D, Baker CA, et al. The role and relevance of phospholipase D1 during growth and dimorphism of Candida albicans. Microbiology. 2001;147(4):879-889.

15. Knechtle P, Goyard S, Brachat S, et al. Phosphatidylinositol-dependent phospholipases C Plc2 and Plc3 of Candida albicans are dispensable for morphogenesis and host-pathogen interaction. Res Microbiol. 2005;156(7):822-829.

16. Odds FC. Candida and candidosis: a review and bibliography $\left(2^{\text {nd }}\right.$ edn $)$ London: Bailliere Tindall pp. 1998;30(5):382-383.

17. Lachke SA, Srikantha T, Tsai LK, et al. Phenotypic switching in Candida glabrata involves phase-specific regulation of the metallothionein gene MT-II and the newly discovered hemolysin gene HLP. Infect Immun 2000;68(2):884-895.

18. Luo G, Samaranayake LP, Cheung BPK, et al. Reverse transcriptase polymerase chain reaction (RT-PCR) detection of HLP gene expression in Candida glabrata and it's possible role in in vitrohaemolysin production. APMIS. 2004;112(4-5):283-290.

19. Tsang CSP, Samaranayake LP. Oral yeasts and coliforms in HIV-infected individuals in Hong Kong. Mycoses. 2000;43(7-8):303-308.

20. Price MF, Wilkinson ID, Gentry LO. Plate method for detection of phospholipase activity in Candida albicans. Sabouraudia. 1982;20(1):714.

21. Staib F. Serum-proteins as nitrogen source for yeast like fungi. Sabouraudia. 1965;4(3):187-193.

22. Manns JM, Mosser DM, Buckley HR. Production of a hemolytic factor by Candida albicans. Infect Immun. 1994;62(11):5154-5156.

23. Luo G, Samaranayake LP, YAU JYY. Candida species exhibit differentia in vitro hemolytic activities. J Clin Microbiol. 2001;39(8):2971-2974.

24. Olaechea PM, Palomar M, Leon-Gil C, et al. Economic impact of Candida infection in the critically ill patients. Eur J Clin Microbiol Infect Dis. 2004;23(4):323-330.

25. Fallon K, Bausch k, Noonan J, et al. Role of aspartic proteases in disseminated Candida albicans infection in mice. Infect Immun. 1997;65(2):551-556

26. Barrett-Bee K, Haynes Y, Watson RG, et al. A comparison of phospholipase activity, cellular adherence and pathogenicity in yeast. $J$ Gen Microbiol. 1985;131(5):1217-1221.

27. Ribeiro MA, Miranda AE, Gambale W, et al. Prevelance and exoenzyme secretion by Candida albicans isolates from oral and vaginal mucosas of HIV-infected women. Mycopathologia. 2004;157(3):255-261.

28. Kadir T, Pisiriciler R, Akyus S, et al. Mycological and cytological examination of oral candidal carriage in diabetic patients and nondiabetic control subjects: thorough analysis of local aetiologic and systemic factors. J Oral Rehabil. 2002;29(5):452-457.

29. Borst A, Fluit AC. High levels of hydrolytic enzymes secreted by Candida albicans isolates involved in respiratory infections. $J \mathrm{Med}$ Microbiol. 2003;52(11):971-974.

30. Mukherjee PK, Chandra J, Kuhn DM, et al. Differential expression of Candida albicans phospholipase B (PLB1) under various environmental and physiological conditions. Microbiology. 2003;149(1):261-267.

31. Samaranayake YH, Dassanayake RS, Cheung BP, et al. Differential phospholipase gene expression by Candida albicans in artificial media and cultured human oral epithelium. APMIS. 2006;114(12):857-866.

32. Kumar CPG, Kumar SSJ, Menon T. Phospholipase and proteinase activities of clinical isolates of Candida albicans from immunocompromised patients. Mycopathologia. 2006;161(4):213-218. 
33. Ollert MW, Wende C, Gorlich M, et al. Increased Expression of Candida albicans Secretory Proteinase, a Putative Virulence Factor, in Isolates from Human Immunodefiency Virus-Positive Patients. J Clin Microbiol. 1995;33(10):2543-2549.

34. De Bernardis F, Chiani P, Ciccozzi M, et al. Elevated aspartyl proteinase secretion and experimental pathogenicity of Candida albicans isolates from oral cavities of subjects infected with human immunodeficiency virus . Infect Immun. 1996;64(2):466-471.

35. Cassone A, Tacconelli E, De Bernardis F, et al. Antiretroviral therapy with protease inhibitors has an early, immune reconstitutionindependent beneficial effect on Candida virulence and oral candidiasis in human immunodeficiency virus-infected subjects. J Infect Dis. 2002;185(2):188-195.
36. De Bernardis F, Sullivan PA, Cassone A. Aspartyl proteinases of Candida albicans and their role in pathogenecity. Med Mycol. 2001;39(4):303313

37. Kantarcioglu AS, Yucel A. Phospholipase and protease activities in clinical candida isolates with reference to the sources of strains. Mycoses. 2002;45(5-6):160-165.

38. Weinberg ED. Iron and infection. Microbiol Rev. 1978;42(1):45-66.

39. Belanger M, Begin C, Jacques M. Lipopolysaccharides of Actinobacillus pluropneumoniae bind pig hemoglobin. Infect Immun. 1995;63(2):656662. 